УДК 534.23

С.А. Найда, д.-р. техн. наук, Д.А. Ляшко

Национальный технический университет Украины “Киевский политехнический институт", ул. Политехническая, 16, г. Киев-56, 03056, Украина.

\title{
Особенности применения акустических фокусирующих линз в пассивной акустотермометрии биологических объектов
}

В работе рассмотрена возможность использования акустических линз для измерения глубинной температуры биологических объектов. Получены аналитические выражения для напряжения шумов от нагретого объекта. Показано, что фокусировка теплового акустического излучения с помощью двояковогнутой эллиптической линзы позволяет измерять и сканировать глубинную абсолютную температуру тела одноканальным, одночастотным акустотермометром. Произведено сравнение эффрективности эллиптической и сорерической линз с одинаковыми параметрами. Библ. 7, рис. 4, табл. 4.

Ключевые слова: фокусировка; ультразвук; акустическая линза; аберрация; пьезоэлектрический приемник; тепловое излучение; биологический объект.

\section{Введение}

В настоящее время ультразвук (УЗ) нашел применение в терапии и хирургии, универсальной литотрипсии (холелитиаз, нефролитиаз, камни желчных протоков, поджелудочной железы и мочеточников, слалотиаз), УЗ диагностике [1].

Интерес к изучению действия УЗ на биологические ткани не ослабевает. Это связано как с естественным опасением возможного риска применение УЗ диагностических систем даже при малых интенсивностях, так и с возможностью вызвать изменения в тканях для достижения терапевтического эффекта, либо управляемого избирательного разрушения в тканях при больших интенсивностях. При этом границы между этими интенсивностями весьма относительны и со временем меняются.

В этой связи привлекает внимание работы К. Корсака (1992), в которой сообщается об обнаружении на поверхности кожи человека особо уязвимых для электрического тока точек и зон, совпадающих с точками акупунктуры. Воздействие на эти точки сфокусированным пучком УЗ волн показано, что болевые рецепторы в них обладают резко выраженным и очень низким порогом возбуждения.
В настоящее время успешно ведутся теоретические и экспериментальные исследования по получению фокусированных УЗ пучков как малой - для УЗ диагностики, так и большой для воздействия на биологические ткани - интенсивности в НТУУ «КПИ» на кафедре акустики и акустоэлектроники [2]. Разработаны оригинальные высокочастотный вольтметр для измерения электрической мощности, подводимой к излучателю, и свободный поплавковый радиометр для измерения фокусируемое акустической мощности. Кроме того, по материалам последних международных выставок медицинской аппаратуры сделан анализ УЗ эхо-скопов и выявлены основные тенденции развития их конструкций.

Анализировались и другие устройства с фокусированным УЗ, в частности, литотрипторы. Это устройства в которых ударная волна длительностью 10 нс создается и фокусируется в задаваемую область с помощью нескольких тысяч пьезоэлементов. При этом, помимо рентгеновской, имеется УЗ локация для фокусировки ударной волны точно на камень.

Таким образом можно выделить следующие перспективные направления исследований: воздействие фокусируемого УЗ различной интенсивности на биологические ткани с динамическим контролем излучаемой мощности, локализация области фрокусировки, коэффицциент затухания УЗ до фокуса с помощью зондирующего УЗ пучка малой интенсивности, определение температуры в фокальной области по шумовому акустическому излучению, принимаемому пьезопреобразователем в промежутках между излучающими мощными импульсами.

Последнее направление представляется наиболее перспективным, т.к. отсутствие безопасного (неинвазивного и пассивного) метода контроля температуры в фокальной области является основным сдерживающим фактором широкого использования фокусируемого УЗ в медицинской практике [3-5].

Целью статьи является получение расчетных соотношений для сферических и эллиптических акустических линз, которые используют- 
ся в акустотермометре с фокусировкой, сравнение эффективности линз различной формы, и анализ влияние этой формы на точность измерения глубинной температуры биологического объекта.

Расчет напряжения шумов от плоского нагретого объекта

Рассматривается объект (рис. 1), находящийся на расстоянии I от пьезоприемника (ПП), который находится в воде в дальней зоне ПП. Дальняя зона $I>\frac{D^{2}}{4 \lambda}(D$ - апертура ПП, $\lambda$ длина УЗ волны в воде (биологическом объекте)) характеризуется тем, что излучение от элементарного источника воспринимается в фразе по всей поверхности ПП.

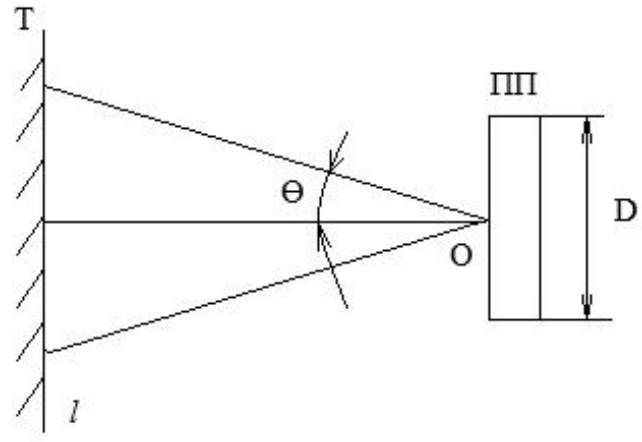

Рис.1. Схема расположения объекта и ПП

Квадрат давления от теплового излучения элементарного источника (сферы) с радиусом $\rho_{K}=\frac{\lambda}{2}$ равен:

$P_{K}^{2}(0)=P_{K}^{2}(I) \cdot\left(\frac{P_{K}}{2}\right)^{2}=P_{K}^{2}(I) \cdot\left(\frac{\lambda}{2 l}\right)$

Размер пятна, образуемого пересечением диаграммы направленности (ДН) с плоскостью объекта, равен $d_{\Pi}=2 \cdot l \cdot \theta$, где $\sin \theta \simeq \theta=\frac{1,62}{\pi} \cdot \frac{\lambda}{D}, 2 \theta=\frac{3,24}{\pi} \cdot \frac{\lambda}{D}=1,03 \frac{\lambda}{D}$. Откуда:

$$
d_{\Pi}=1,03 \cdot l \cdot \frac{\lambda}{D}
$$

Суммарный квадрат давления от некоррелированных источников в пятне $P_{\Sigma}^{2}(0)=N \cdot P_{\mathrm{K}}^{2}(I) \cdot\left(\frac{\lambda}{2 l}\right)^{2}$, где $N=\left(\frac{d_{\Pi}}{\lambda}\right)^{2}=\left(\frac{l}{D}\right)^{2}$, или $P_{\Sigma}^{2}(0)=P_{\kappa}^{2}(I) \cdot\left(\frac{\lambda}{2 D}\right)^{2}$, или
$P_{\Sigma}^{2}(0)=\frac{\pi^{2}}{4} \cdot \frac{z_{2}}{A_{0}} \cdot k_{\sigma} \cdot T \cdot \Delta f$

где $A_{0}$ - площадь рабочей поверхности ПП, $\Delta f$ полоса пропускания ПП, $k_{\sigma}=1,38 \cdot 10^{-23}$ Дж/К постоянная Больцмана, $z_{2}$ - акустический импеданс воды, Т - абсолютная температура объекта.

Среднее во времени и по поверхности давление от источников в ближней зоне оказывается в $\pi^{2}$ раз меньше, чем от источников в дальней зоне.

Например, для ПП с $D=28$ мм и работающего на частоте $f \simeq 1 \mathrm{MГц}(\lambda=1,5 \mathrm{~mm})$ : $\mathrm{z}_{б}=\frac{D^{2}}{4 \cdot \lambda}=120 \mathrm{MM}$; $D=14 \mathrm{~mm}: z_{\sigma}=30 \mathrm{~mm}$.

Следовательно, если длина пути УЗ волны в воде в направлении оси ПП гораздо меньше $z_{б}$, TO

$$
U_{\text {из }}^{2} \ll \frac{z_{2}}{A_{0}} \cdot k_{б} \cdot T \cdot K_{\Pi 0}^{2} \cdot \Delta f
$$

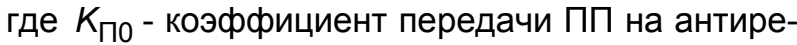
зонансной частоте. Квадрат напряжения собственных шумов ПП $U_{\text {Пп }}^{2}$ определяется только $z_{2}$ и от длины не зависит. Поэтому в ближней зоне $\frac{U_{\text {из }}^{2}}{U_{\text {Пп }}^{2}} \ll 1$, и для фокусируемого ПП:

$$
U_{\Sigma}^{2}=U_{\Pi \Pi}^{2}\left[1+\left(\frac{\pi \cdot D}{2 F}\right)^{2}\right],
$$

где F- фокусное расстояние ПП. При $\left(\frac{\pi \cdot D}{2 F}\right)^{2}=1,44: \frac{U_{\Sigma}^{2}}{U_{\Pi \Pi}^{2}}=2,44$.

Реализовать фокусирование УЗ волн в акустотермометре можно несколькими способами: сферическими пьезоэлементами, акустическими линзами, кольцевыми решетками пьезоэлементов. Наиболее приемлемым из них является использование акустических линз, поскольку они защищают пьезоэлемент от воздействия жидкости и просты в изготовлении. Рассмотрим особенности применения акустических линз для акустотермометрии, а именно, для фрокусирования слабых шумовых сигналов в режиме приемa. 


\section{Расчет двояковогнутой эллиптической аку-} стической линзы

Как известно [1], вогнутые акустические линзы должны изготавливаться из материала с показателем преломления $n=\frac{c_{ж}}{c_{л}}<1$.

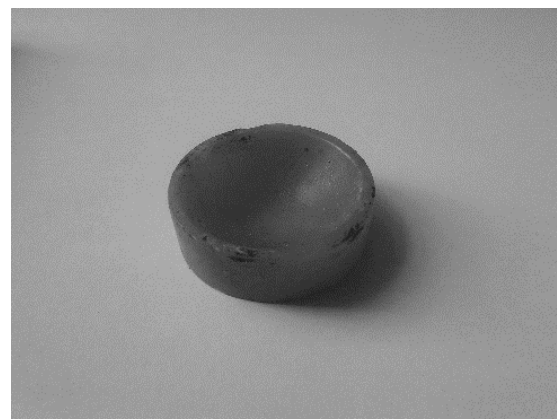

a)
В качестве такового выберем полистирол (рис.2,a), который легко обрабатывается, и из которого линзу можно изготовить с помощью пресс-фрормы (рис.2,б), что и было сделано авторами.

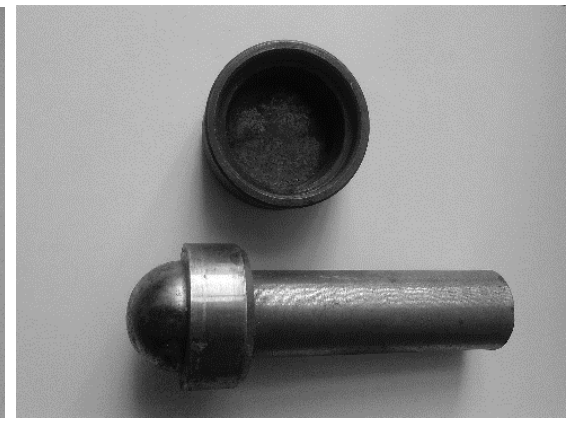

б)

Рис.2. Изображение акустической линзы из полистирола (а) и пресса-формы для ее изготовления (б)

Таким образом, исходные данные для расчета следующие: $c_{\text {пол }}=2,3 \cdot 10^{3} \mathrm{~m} / \mathrm{c}$-скорость УЗ волн в полистироле; $c_{\mathrm{B}}=1,5 \cdot 10^{3} \mathrm{M} / \mathrm{C}$ - скорость УЗ волн в воде (биологической ткани), $n=0,635, D=28$ мм .

Уравнение поверхности линзы [1]: $x^{2}-2 F \cdot(1-n) \cdot Z+\left(1-n^{2}\right) \cdot Z^{2}=0$.

После подстановки в (6) $x=\frac{D}{2}, z=z_{\text {макс }}$ и
$F=4 \cdot z_{\text {макс }}$, получаем:

$z_{\text {макс }}=\frac{D}{2 \sqrt{(7-n)(1-n)}}=9,15 \mathrm{Mm}$,

$F \simeq 37$.

Уравнение (6) приводится к виду:

$x=\sqrt{26,82 \cdot z-0,597 \cdot z^{2}} \cdot \lg (z)$.

Результаты расчета формы поверхности вогнутой акустической линзы приведены в табл.1.

Таблица 1. Форма поверхности вогнутой акустической линзы

\begin{tabular}{|c|c|c|c|c|c|c|c|c|c|c|}
\hline$z$, мM & 0,25 & 1,00 & 2,00 & 3,00 & 4,00 & 5,00 & 6,00 & 7,00 & 8,00 & 9,00 \\
\hline X, Мм & 2,58 & 5,12 & 7,16 & 8,67 & 9,89 & 10,92 & 11,81 & 12,59 & 13,28 & 14,00 \\
\hline
\end{tabular}

Полученная поверхность близка к сферической с $R=15 \mathrm{мм}: x^{2}+(Z-R)^{2}=R^{2}$.

Изготовленная по данным табл.1 линза имеет следующие размеры (рис. 3).

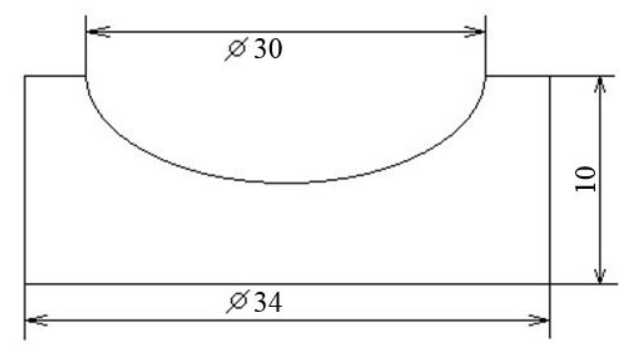

Рис. 3. Изображение плосковогнутой акустической линзы для акустотермометра
Такая линза может быть использована как плосковогнутая с $F \simeq 37 \mathrm{~mm},\left(\frac{\pi \cdot D}{2 F}\right)^{2}=1,6$.

Интенсивность шума с линзой должна быть в 1,8 раза, и $U_{\Sigma}$ в $\sqrt{1,8}=1,35$ раз больше, чем с тем же ПП без линзы. Этого достаточно, чтобы проверить основное соотношение о фокусировке линзой теплового акустического шума без модуляции - простым удалением линзы.

В случае двояковогнутой линзы: $\left(\frac{\pi \cdot D}{2 F}\right)^{2}=4 \cdot 1,4=5,6$, и указанное выше отношение увеличится до значения 3,8 . 
В действительности же получится меньше значение, т.к. диаметр преобразователя 28мм . Оценить, во сколько раз, можно, взяв $D=24 \mathrm{mм}$; тогда $\left(\frac{\pi \cdot D}{2 F}\right)^{2}=1,2:((2+4 \cdot 1,2) / 2)=3,4$.

Об изменении свойств эллиптической линзы при отклонении показателя преломления от принятого при расчете поверхности линзы

Уравнения сечения поверхности линзы плоскостью $x 0 z$ имеет вид (6). Преобразуем его к каноническому виду, выделив полный квадрат из зависящих от $z$ членов:

$$
\begin{aligned}
& \left(1-n^{2}\right)\left(\mathrm{z}^{2}-2 \mathrm{~F} \frac{1-n}{1-n^{2}} \mathrm{z}+\mathrm{F}^{2} \frac{1}{(1+n)^{2}}-\right. \\
& \left.-\mathrm{F}^{2} \frac{1}{(1+n)^{2}}\right)+x^{2}=0 \\
& \left(1-n^{2}\right)\left(\mathrm{z}-\frac{\mathrm{F}}{1+n}\right)^{2}-\frac{1-n^{2}}{(1+n)^{2}} \mathrm{~F}^{2}+x^{2}=0 .
\end{aligned}
$$

Введём новую переменную:

$$
z^{\prime}=z-\frac{F}{1+n},
$$

тогда

$$
\frac{x^{2}}{b^{2}}+\frac{z^{\prime 2}}{a^{2}}=1,
$$

где $a^{2}=\frac{F^{2}}{(1+\mathrm{n})^{2}}, b^{2}=\frac{1-n}{1+n} F^{2}$.

Из (6) следует:

$F=\frac{x^{2}+\left(1-n^{2}\right) \cdot z^{2}}{2 \cdot z \cdot(1-n)}=\frac{(D / 2)^{2}+\left(1-n^{2}\right) \cdot z_{\text {макс }}^{2}}{2 \cdot z_{\text {макс }}(1-n)}$

т.е. при заданной функции $x(z)$ в форме эллипса, определяется параметром $n$.

Смещение фокусного расстояния в сторону сферической линзы в зависимости от апертуры УЗ пучка

Будем считать, что заданы $n, D, F_{0}\left(F_{0}\right.$ - фокусное расстояние для центральных лучей).

Для сфрерической вогнутой линзы:

$$
F_{0}=\frac{R_{\text {Л }}}{1-n},
$$

$D / 2 \leq R_{\text {л }}, R_{\text {л }}$ - радиус кривизны линзы.

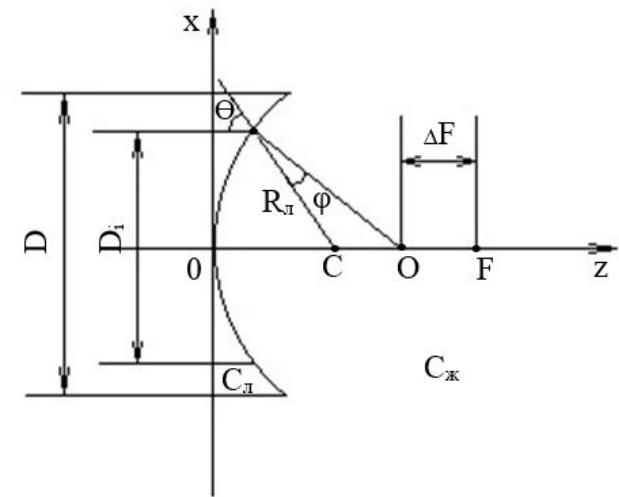

Рис. 4. Изображение смещения фокусного расстояния $\Delta F$

Расчетная формула для величины смещения (рис. 4):

$$
\Delta F=R\left[\frac{1}{1-n}-\left(1+\frac{1}{\frac{\cos \varphi}{n}-\cos \varphi}\right)\right],
$$

где

$\sin \theta=\frac{D}{2 \cdot R_{\text {Л }}}<1, \sin \varphi=\mathrm{n} \cdot \sin \theta$.

Отклонение поверхности эллиптической линзы от поверхности сферической в зависимости от $z$ найдем при одинаковом фокусном расстоянии.

Уравнение кривой осевого сечения поверхности эллиптической линзы при условии:

$$
\begin{aligned}
& F=4 z_{\text {макс }}=\frac{2 D}{\sqrt{(z-n)(1-n)}}, \\
& x^{2}-\frac{4 D(1-n)}{\sqrt{(z-n)(1-n)}} z+\left(1-n^{2}\right) z^{2}=0,
\end{aligned}
$$

$$
x_{\text {эллипт }} \simeq \sqrt{2 z R_{л}}\left(1-\frac{\left(1-n^{2}\right) \cdot z}{4 R_{л}}\right) .
$$

Уравнение этой же линии сферической линзы: $x_{\text {сф }}^{2}+\left(z-R_{л}\right)^{2}=R_{л}^{2}$, откуда

$x_{\text {cक }} \simeq \sqrt{2 z \cdot R_{\text {Л }}}\left(1-\frac{z}{4 R_{\text {Л }}}\right)$,

где $R_{\text {л }}$ определяется из выражения (13).

Следовательно,

$\Delta x=x_{\text {эллипт }}-x_{\text {сф }} \simeq \sqrt{\frac{n^{4}}{8 \mathrm{R}_{\text {л }}}} \cdot z^{3 / 2}$. 
Для случая: $n=0,59, F=36,5 \mathrm{~mm}, \mathrm{R}_{л}=15 \mathrm{~mm}$, согласно $(20), \quad \Delta x=4,48 \cdot 10^{-2} \cdot z^{3 / 2}$, и результаты расчетов приведены в табл.2.

Таблица 2. Отклонение поверхности эллиптической линзы от сферической поверхности

\begin{tabular}{|c|c|c|c|c|c|c|c|c|c|}
\hline$z, \mathrm{MM}$ & 1 & 2 & 3 & 4 & 5 & 6 & 7 & 8 & 9 \\
\hline$\Delta x, \mathrm{MM}$ & 0,045 & 0,127 & 0,232 & $0,368 \simeq \lambda / 2$ & 0,5 & 0,66 & 0,83 & 1,02 & 1,2 \\
\hline$x_{\mathrm{Cq},}, \mathrm{MM}$ & 5,4 & 7,5 & 9 & 10,2 & 11,3 & 12 & 12,7 & 13,3 & 13,8 \\
\hline
\end{tabular}

Вычислим также по формуле (14) смещение фокуса в сторону сферической линзы для $n=0,635, F=37$ мм . Определяем по (13): $R_{\text {л }}=13,51 \mathrm{~mm} ; \frac{1}{1-n}=2,74$, и сводим результаты в табл.3.

Таблица 3. Смещение фокусного расстояния

\begin{tabular}{|c|c|c|c|c|c|c|c|c|c|c|c|c|}
\hline$D_{i}, \mathrm{MM}$ & 2 & 4 & 6 & 8 & 10 & 12 & 14 & 16 & 18 & 20 & 22 & 26 \\
\hline$\Delta F, \mathrm{MM}$ & 0,0446 & 0,168 & 0,375 & 0,669 & 1,05 & 1,54 & 2,12 & 2,83 & 3,67 & 4,68 & 5,9 & 9,63 \\
\hline
\end{tabular}

Скоррелированными являются лучи, выходящие из области с $D_{\text {кор }}=9 \mathrm{Mm}$, $\frac{\pi \cdot D_{\text {кор }}}{2 F}=0,145, \quad(2+0,145) / 2=1,08$, тогда как для эллиптической линзы с $D=28 \mathrm{~mm}$ : $\left(\frac{\pi \cdot D^{2}}{2 F}\right)=1,7$.

Сравнение эллиптической и сорерической линзы при использовании в акустотермометре

Обычно фокусирующие линзы применяются при фрокусировке когерентных узкополосных излучений $[6,7]$.
Фокусировка широкополосного излучения в У3 эхо-скопах осуществляется с помощью кольцевых или линейный матриц пьезоэлементов, на которые импульс излучения подается со сдвигом по времени, соответствующим эллиптической линзе.

От эллиптической сферическая линза отличается тем, что точка фокуса луча зависит от его расстояния до оси линзы: по мере его увеличения (табл.3) она приближается к линзе.

Проведем по формуле (14) аналогичные расчеты для сфрерической линзы с $D=20$ мм, $\mathrm{F}=30 \mathrm{mм}$ из оргстекла $(n=0,56), R_{л}=13,2 \mathrm{мm}$ (табл. 4).

Таблица 4. Смещение фокусного расстояния для линзы из оргстекла

\begin{tabular}{|l|l|l|l|l|l|l|l|l|l|l|}
\hline$D_{i}$, Мм & 2 & 4 & 6 & 8 & 10 & 12 & 14 & 16 & 18 & 20 \\
\hline$\Delta F$, мM & 0 & 0,073 & 0,21 & 0,4 & 0,66 & 0,98 & 1,37 & 1,84 & 24 & 30,8 \\
\hline
\end{tabular}

Средняя частота полосы электрического шума с электродов пьезопреобразователя $f_{0}=2 \mathrm{MГц} \mathrm{.}$

Поскольку радиус корреляции $\rho_{\mathrm{K}}=\lambda / 2$, то $D_{i}=8=D_{\kappa}$ - диаметр области, в которой шумовое акустическое излучение из области фокуса находится в фразе

$$
\begin{aligned}
& U_{\text {пп }}^{2}=\left(\frac{\rho_{\mathrm{K}}}{F}\right)^{2} \cdot p_{F}^{2} \cdot K_{\Pi 0}^{2} \cdot \frac{D_{\mathrm{K}}^{2}}{D^{2}}= \\
& =\left(\frac{\pi D_{\mathrm{K}}}{2 F}\right)^{2} \frac{z}{A_{0}} \cdot k_{\sigma} \cdot T \cdot K_{\Pi 0}^{2} \cdot \Delta f=\left(\frac{\pi D_{\mathrm{K}}}{2 F}\right)^{2} \cdot U_{\mathrm{a \kappa}}^{2}
\end{aligned}
$$

Суммарный квадрат напряжения на ПП равен:

$U_{\Sigma \Pi \Pi}^{2}=U_{\mathrm{a \kappa}}^{2}\left[2+\left(\frac{\pi D_{\mathrm{K}}}{2 F}\right)^{2}\right]$ 


\section{Выводы}

Из рассмотренных в работе различных способов фрокусировки следует, что наибольшее значение $(D / 2 F)$ получается с двояковогнутой линзой.

В случае линзы из оргстекла $(\pi D / 2 F)^{2}$ на $25 \%$ больше, чем для линзы из полистирола. При этом существенно, чтобы линза была безаберрационной, т.е. эллиптической.

$$
\text { При }\left(\frac{\pi D_{\kappa}}{2 F}\right)^{2}=0,175 \ll 2 \text {, поэтому точность }
$$

определения яркостной температуры тела $\theta^{\prime}$ определяется шумовой компонентой $2 U_{\text {ак }}^{2}$, а точность определения глубинной температуры по корреляционной добавке $0,175 \cdot \theta_{\text {к }}=\theta^{\prime}$, откуда $\theta_{\kappa}=5,7 \cdot \theta^{\prime}$. В случае $\left(\frac{\pi D}{2 F}\right)^{2}=1,6$ точность определения глубинной температуры будет определятся суммарным шумом $U_{\Sigma}^{2}$ пп и отношением $1,6 / 3,2=0,5$, т.е. в 2 раза хуже. При $\left(\frac{\pi D_{\kappa}}{2 F}\right)^{2} \gg 2$ точность равна пороговой.

\section{Список использованных источников}

1. Дідковський В.C., Найда C.A. П'єзоелектричні перетворювачі медичних ультразвукових сканерів: Навч. посібник. -К.: НМЦВО, 2000. -178с.

2. Дидковский В.С., Найда С.А. Актуальные направления исследований в области технической акустики // Электроника и связь. 1998. -Ч.3, -№4. -С.608-612.

3. Bowen T. Acoustic passive remote temperature sensing // Acoustic imaging. -1982. -P.549-561.

4. Найда С.А. Акустотермометрия жидких объектов с помощью пьезоприемников мегагерцевого диапазона //Техническая диагностика и неразрушающий контроль. -2002, №3. C.41-48.

5. Найда C.A. Пьезоприемники теплового акустического излучения для зондирования внутренней температуры объектов //Техническая диагностика и неразрушающий контроль. -2003, №1. - С. 25-29.

6. Смарышев М.Д. Направленность гидроакустических антенн. - Л.: Судостроение. 1973. -278 c.

7. Справочник по гидроакустике / А.П. Евтютов, А.Е. Колесников, А.П. Ляликов и др. -Л.: Судостроение. -1982. -344 с.

Поступила в редакцию 21 апреля 2015 г.

\section{УДК 534.23}

\section{С.А. Найда, д.-р. техн. наук, Д.О. Ляшко}

Національний технічний університет України «Київський політехнічний інститут», ул. Політехнічна, 16, м. Київ-56, 03056, Україна.

\section{Особливості застосування акустичних фокусуючих лінз в пасив- ній акустотермометрії біологічних об'єктів}

В роботі розглянута можливість використання акустичних лінз для вимірювання глибинної температури біологічних об'єктів. Отримано аналітичні вирази для напруги шумів від нагрітого об'єкта. Показано, що фокусування теплового акустичного випромінювання за допомогою двояковгнутої еліптичної лінзи дозволяє вимірювати і сканувати глибинну абсолютну температуру тіла одноканальним, одночастотним акустотермометром. Здійснено порівняння ефрективності еліптичної і сореричної лінз з однаковими параметрами. Бібл. 7, рис. 4, табл. 4.

Ключові слова: фокусировка; ультразвук; акустична лінза; аберрація; п'езоелектричний приймач; теплове випромінювання; біологічний об'єкт. 
UDC 534.23

S.A. Naida, Dr.Sc., D.A. Lyashko

National Technical University of Ukraine "Kiev Polytechnic Institute",

st. Polytechnique, 16, Kiev-56, 03056, Ukraine.

\section{Features of Application of the Acoustic Focusing Lenses in Passive Acoustothermometry of Biological Objects}

The paper considers the possibility of using the acoustic lens for measuring the internal temperature of biological objects. The analytical equations for the noise voltage from the heated object had been received. It is shown that the focus of the thermal acoustic emission with the help of biconcave elliptic lens allows us to measure and scan the deep absolute body temperature by the single-channel and single frequency acoustothermometer. The comparison of the effectiveness of elliptical and spherical lenses with the same parameters had been done. References 7 , figures 4 , tables 4 .

Keywords: focus; ultrasound; acoustic lens; aberration; a piezoelectric receiver; thermal acoustic emission; biological object.

\section{References}

1. Didkovskiy V.S., Naida S.A. (2000), Piezoelectric converters of medical ultrasound scanners. (NMTSVO, Kyiv). (Ukr)

2. Didkovskiy V.S. Naida S.A. (1998), "Topical avenues of research in the field of technical acoustics". Electronics and Communications. Vol. 4, No.3, pp. 608-612. (Rus)

3. Bowen T. (1982), "Acoustic passive remote temperature sensing". Acoustic imaging. Vol. 11, pp.549561.

4. Naida S.A. (2002), "Acoustic thermometry of liquid objects using piezoelectric sensors of megahertz range". Technical diagnostics and non-destructive testing. Vol. 3, pp.41-48. (Rus)

5. Naida S.A. (2003), "Piezoreceivers of thermal acoustic emission for probing the core temperature of objects". Technical diagnostics and non-destructive testing. Vol. 1, pp.25-29. (Rus)

6. Smaryshev M.D. (1973), Directivity of hydro-acoustic antennas. (Shipbuilding, Leningrad). (Rus)

7. Evtyutov A.P., Kolesnikov A.E., Lyalikov A.P. etc. (1982), Reference manual for hydroacoustics. (Shipbuilding, Leningrad). (Rus) 